\title{
Connective tissue disease-associated pulmonary arterial hypertension in Chinese patients
}

\author{
Yan-Jie Hao' ${ }^{1}$ Xin Jiang ${ }^{2}$, Wei Zhou' ${ }^{1}$, Yong Wang ${ }^{3}$, Lan Gao' ${ }^{1}$ Yu Wang ${ }^{1}$, \\ Guang-Tao Li ${ }^{1}$, Tao Hong ${ }^{4}$, Yong Huo ${ }^{4}$, Zhi-Cheng Jing ${ }^{2}$ and Zhuo-Li Zhang ${ }^{1}$
}

Affiliations: 'Dept of Rheumatology and Clinical Immunology, Peking University First Hospital, Beijing, China. ${ }^{2}$ Thrombosis and Vascular Medicine Center, State Key Lab of Cardiovascular disease, Fu Wai Hospital, Peking Union Medical College and Chinese Academy of Medical Science, Beijing, China. ${ }^{3}$ Dept of Pulmonary Vascular disease, Beijing Shijitan Hospital, Capital Medical University, Beijing, China. ${ }^{4}$ Dept of Cardiology, Peking University First Hospital, Beijing, China.

Correspondence: Zhuo-Li Zhang, Dept of Rheumatology and Clinical Immunology, Peking University First Hospital, 8 Xishiku St, Beijing 100034, PR China. E-mail: zhuoli.zhangR126.com

ABSTRACT We sought to investigate the characteristics, survival and risk factors for mortality in Chinese patients with connective tissue disease (CTD)-associated pulmonary arterial hypertension (APAH) in modern therapy era.

129 consecutive adult patients who visited one of three referral centres in China with a diagnosis of CTDAPAH confirmed by right heart catheterisation during the previous 5 years were enrolled. The end-point was all-cause death or data censoring.

Systemic lupus erythematosus was the most common underlying CTD (49\%) and systemic sclerosis just accounted for $6 \%$ in this cohort. The overall survival at 1 and 3 years was $92 \%$ and $80 \%$, respectively. Pericardial effusion, a shorter 6-min walk distance, lower mixed venous oxygen saturation, higher pulmonary vascular resistance (PVR) and alkaline phosphatase (ALP), and lower total cholesterol levels were all associated with a higher risk of death among the study population. Higher PVR and ALP were independent predictors of mortality.

In conclusion, unlike in western patients, systemic lupus erythematosus is the most common underlying disease in Chinese patients with CTD-APAH. The survival of Chinese patients with CTD-APAH in the modern treatment era is similar to that in western countries. Elevated PVR and ALP are independent risk factors for poor outcomes.

@ERSpublications

Systemic lupus erythematosus is the most common underlying disease in Chinese patients with CTD-APAH http://ow.ly/vo4x6

\footnotetext{
For editorial comments see page 839.

Received: Oct 202013 | Accepted after revision: March 262014 | First published online: May 022014

Conflict of interest: None declared.

Copyright @ERS 2014
} 


\section{Introduction}

Pulmonary arterial hypertension (PAH) is a severe complication of connective tissue disease (CTD), with a poor prognosis. Fortunately, the emergence of targeted therapies has improved the outcome for PAH patients in the modern treatment era. Several studies from USA and Europe [1,2] have investigated the clinical features and outcomes of PAH patients secondary to CTD. They found that systemic sclerosis (SSc)-associated PAH (APAH) was the most common type of CTD-APAH in Caucasians, and the estimated 1- and 3-year survival rates of these patients were about $80 \%$ and $50 \%$, respectively, in the modern treatment era.

During the past decades, ethnic disparities in the prevalence of some CTDs have been reported. For instance, Chinese persons have a higher prevalence of systemic lupus erythematosus (SLE) than Caucasians [3-5], but SSc is less prevalent in East Asia than in Europe, Australia and North America [6-9]. Because of these disparities, it is not known whether the characteristics of Chinese patients with CTD-APAH are different from those of Caucasians. Additionally, with the increasing use of targeted therapies for PAH in China, it is not known whether these medications have improved the clinical outcomes of Chinese patients. To address these questions, we initiated a research project with the aim of investigating baseline characteristics, survival and risk factors for mortality in Chinese patients with CTD-APAH, and comparing the clinical features and survival of patients with different types of CTD-APAH.

\section{Methods}

Patients

The study was conducted in three national referral centres for $\mathrm{PAH}$ in China. All consecutive adult $(\geqslant 18$ years of age) CTD patients who visited any of the three centres with an established diagnosis of PAH confirmed by right heart catheterisation (RHC) for the first time between July 2006 and May 2011 were enrolled into our database. Patients' clinical data at the time of establishing the PAH diagnosis before administration of any targeted therapies were recorded.

\section{Inclusion and exclusion criteria}

The inclusion haemodynamic criteria for PAH at RHC were mean pulmonary artery pressure (mPAP) $\geqslant 25 \mathrm{mmHg}$ at rest, pulmonary capillary wedge pressure $\leqslant 15 \mathrm{mmHg}$, and pulmonary vascular resistance $(P V R) \geqslant 3$ Wood units [10]. The classification criteria for the various CTDs applied were the 1997 American Rheumatism Association (ACR) criteria for SLE [11, 12], 2002 international classification criteria for primary Sjögren syndrome (pSS) [13], 1990 ACR classification criteria for Takayasu arteritis (TA) [14], 1987 Sharp criteria for mixed connective tissue disease (MCTD) [15] and 1980 ACR classification criteria for SSc [16]. The diagnoses of CTDs were verified by experienced rheumatologists.

Patients with a definite history of idiopathic PAH, chronic obstructive pulmonary disease, obstructive sleep apnoea, other pulmonary diseases with mixed restrictive or obstructive patterns, chronic thromboembolism, left heart disease, portal hypertension, HIV infection, drug and toxin exposure, or any other diseases known to be associated with pulmonary hypertension $(\mathrm{PH})$ were excluded.

CTD patients who fulfilled the haemodynamic inclusion criteria but had moderate interstitial lung disease (ILD) on high-resolution computed tomography (HRCT) (one- to two-thirds of the lung field involved) combined with a total lung capacity $<60 \%$ predicted on pulmonary function testing, or severe ILD on HRCT (more than two-thirds of the lung field involved) were defined as having ILD-associated PH (ILD$\mathrm{PH})[1,2]$. Although ILD-PH patients were excluded from our CTD-APAH cohort, the survival of these patients was compared with that of CTD-APAH patients.

\section{Treatments and follow-up}

After treatment commenced, patients were followed-up regularly every 3-6 months and RHC was repeated every 6-12 months depending on the clinical need. In cases of positive acute vasodilator testing [10], a calcium channel blocker (CCB), most commonly diltiazem, was initiated. If no improvement was observed after 3-6 months of CCB (defined as $\geqslant 20 \%$ fall in 6-min walk distance (6MWD), an increase in World Health Organization functional class (WHO FC), or $\geqslant 20 \%$ increase in mPAP and PVR), PAH-targeted monotherapy was instituted, including endothelin receptor antagonists, prostanoids or phosphodiesterase inhibitors. In cases of negative acute vasodilator testing, PAH-targeted monotherapies were selected directly. Combination PAH-targeted therapies were initiated if disease did not improve after 3-6 months of monotherapy. If necessary, basic treatments for PAH including diuretics, digoxin, oxygen or warfarin were given. Glucocorticoids and immunosuppressants were administered when required for the underlying CTD rather than $\mathrm{PAH}$ itself.

The follow-up period for analysis of survival data ended in June 2011. The date of death, which was determined by telephone if the death happened outside of the hospital, was recorded. The duration of 
follow-up was defined as the time from the first diagnosis by RHC until death or known status at the last follow-up.

\section{Statistical analysis}

Continuous variables are presented as mean \pm SD (for parametric data) or median (interquartile range) (for nonparametric data), whereas categorical variables are presented as $\mathrm{n}(\%)$. Comparisons of baseline data were performed between patients with SLE-APAH and non-SLE-APAH, and between patients with TA-APAH and non-TA-APAH. Continuous variables were compared by use of t-tests (for parametric data) or MannWhitney U-tests (for nonparametric data). Categorical variables were compared using Chi-squared tests.

Survival analysis was performed using the Kaplan-Meier method with comparisons performed by log-rank test. The primary end-point was all-cause death or data censoring. Univariate and multivariate Cox proportional hazards models were performed to determine the variables associated with an increased risk of death. The potential predictive factors investigated in the Cox model were age, sex, CTD type, WHO FC, $6 \mathrm{MWD}$, right ventricular diastolic diameter, pericardial effusion, forced vital capacity (FVC) \% predicted, diffusing capacity of the lung for carbon monoxide (DLCO) \% predicted, FVC \% predicted/DLCO \% predicted ratio, mean right atrial pressure, mPAP, cardiac index, PVR, mixed venous oxygen saturation $\left(\mathrm{SvO}_{2}\right)$, various biochemical parameters, autoantibodies, and use of $\mathrm{PAH}$-targeted therapies, glucocorticoids and immunosuppressants. A two-tailed p-value $<0.05$ was used to indicate statistically significant differences. All computations were performed using the SPSS 13.0 statistical software (IBM, Armonk, NY, USA).

\section{Results}

\section{Baseline characteristics}

A total of 144 CTD patients were diagnosed with PAH at the three referral centres between July 2006 and May 2011. After excluding 15 patients with ILD, 129 patients were eventually included in our study. Their mean $\pm \mathrm{SD}$ age was $39.3 \pm 12.3$ years (range 18-71 years) and there was a marked female predominance (92.2\%). The median duration from the presence of PAH-associated symptoms to catheterisation for diagnostic purposes was 15.1 months (range 0.4-239.9 months). SLE was the leading underlying CTD (49\% of the patients), while other underlying CTDs, in a descending order, were pSS (16\%), TA (12\%), MCTD (9\%) and SSc (6\%). Other less common CTDs included rheumatoid arthritis, polymyositis/dermatomyositis, adult-onset Still's disease, undifferentiated CTD and primary antiphospholipid syndrome (fig. 1).

At the time of establishing the diagnosis of PAH, the mean 6MWD was $380.6 \pm 100.9 \mathrm{~m}$ and $56.6 \%$ of the patients were in WHO FC III/IV. Pericardial effusion was detected in $24.8 \%$ and right ventricular enlargement in 57\%. mPAP was $51.1 \pm 14.3 \mathrm{mmHg}$, median PVR was 10.6 Wood units and mean cardiac index was $2.7 \pm 0.9 \mathrm{~L} \cdot \mathrm{min}^{-1} \cdot \mathrm{m}^{-2}$. Only five $(3.5 \%)$ patients (two with TA, one SLE, one MCTD and one undifferentiated CTD) showed a positive acute vasodilator response. There were no differences in positive response rates among the different CTD subgroups.

PAH-targeted medications were administered to $110(85.3 \%)$ patients, including sildenafil in $33.3 \%$, vardenafil in $23.6 \%$, beraprost in $23.6 \%$, bosentan in $16.7 \%$ and inhaled iloprost in $13.2 \%$; $36(32.7 \%)$ of the 110 patients received combination therapies with two of these drugs. Almost $90 \%$ of patients received glucocorticoids, and $45 \%$ received immunosuppressants, including cyclophosphamide, cyclosporine, azathioprine, mycophenolate mofetil or methotrexate. Cyclophosphamide was most often used (67.2\% of these patients).

The baseline demographics, clinical characteristics, and cardiac and pulmonary investigations of all patients and the three main CTD subgroups are summarised in tables 1 and 2.

\section{Comparisons between SLE-APAH and non-SLE-APAH subgroups}

In our cohort, 62 patients had SLE-APAH and 67 had non-SLE-APAH. Compared with the non-SLE-APAH group, the SLE-APAH patients were older and more often female. The percentage of anti-U1 ribonucleoprotein positivity was higher in the SLE-APAH group than the non-SLE-APAH group $(54.0 \%$ versus $29.7 \%, \mathrm{p}<0.01$ ) and the percentage of patients receiving glucocorticoids was also higher in the SLEAPAH group than the non-SLE-APAH group (98.4\% versus $80.6 \%, \mathrm{p}<0.01$ ). However, there were no significant differences in functional status, echocardiography findings, or haemodynamic and biochemical parameters between the two subgroups.

\section{Comparisons between TA-APAH and non-TA-APAH subgroups}

Comparisons between the TA-APAH and non-TA-APAH subgroups revealed no significant differences in age or sex, but the disease duration from the onset of $\mathrm{PAH}$-associated symptoms to the diagnosis of PAH was significantly longer in the TA-APAH group than the non-TA-APAH group (median (interquartile 


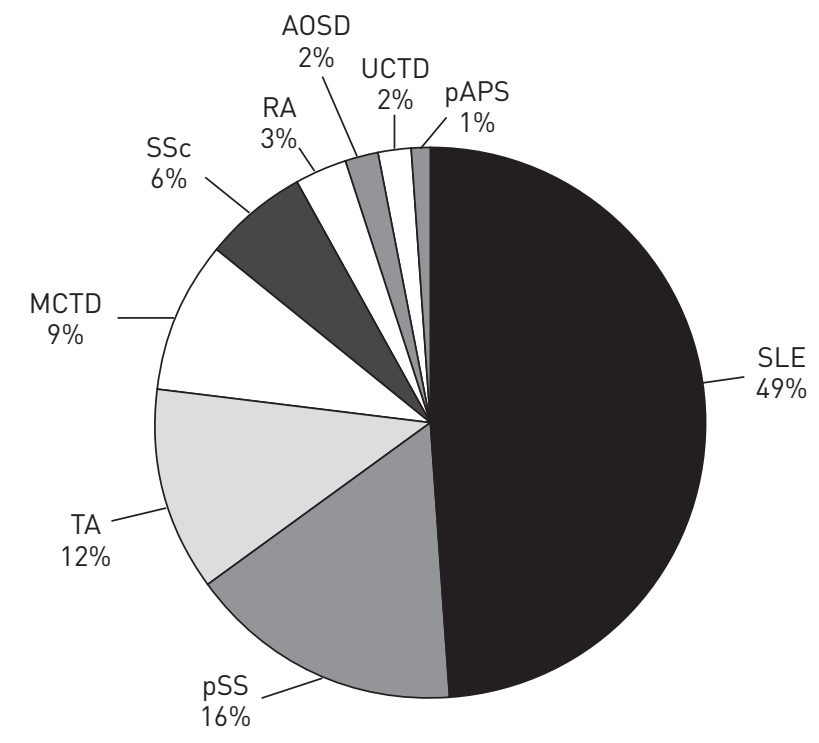

FIGURE 1 Proportions of different underlying connective tissue diseases (CTDs) in 129 patients with CTD-associated pulmonary arterial hypertension (APAH). Systemic lupus erythematosus (SLE) was the most common underlying type of CTD $(n=62,49 \%)$, while only $6 \%$ of patients had systemic sclerosis (SSc)-APAH. pSS: primary Sjögren syndrome; TA: Takayasu arteritis; MCTD: mixed connective tissue disease; RA: rheumatoid arthritis; AOSD: adult-onset Still's disease; UCTD: undifferentiated connective tissue disease; pAPS: primary antiphospholipid syndrome.

range) $54.0(16.0-110.8)$ versus $12.6(6.0-36.0)$ months, $\mathrm{p}<0.001)$. It was notable that systolic pulmonary arterial pressure (sPAP) was markedly higher in TA-APAH patients than non-TA-APAH patients $(95.3 \pm 22.6$ versus $85.5 \pm 23.7 \mathrm{mmHg}, \mathrm{p}<0.05)$; however, diastolic pulmonary arterial pressure (dPAP) was lower in TA-APAH patients $(19.4 \pm 9.4$ versus $32.8 \pm 11.6 \mathrm{mmHg}, \mathrm{p}<0.001)$.

\section{TABLE 1 Baseline demographic and clinical characteristics of all patients and three connective tissue disease subgroups}

\section{Patients $n$}

Age years

Females

Duration from symptoms

onset to diagnosis" months

WHO FC III/IV

6MWD $\mathrm{m}$

$\mathrm{SCr}^{\oplus} \mu \mathrm{mol} \cdot \mathrm{L}^{-1}$

$\mathrm{UA}^{+} \mu \mathrm{mol} \cdot \mathrm{L}^{-1}$

ALP ${ }^{\S} \mathrm{IU} \cdot \mathrm{L}^{-1}$

TBil $^{f} \mu \mathrm{mol} \cdot \mathrm{L}^{-1}$

$\mathrm{TC}^{\# \#} \mathrm{mmol} \cdot \mathrm{L}^{-1}$

Anti-U1 RNP

PAH-targeted therapy

Glucocorticoids

Immunosuppressants

\section{All patients}

SLE-APAH

62

$37.2 \pm 12.2$

$61(98.4)$

$119(92.2)$

$15.1(6.4-39.7)$

$73(56.6)$

$380.6 \pm 100.9$

$63.7 \pm 20.6$

$354.8 \pm 130.7$

70.0 (50.0-100.0)

$13.0(9.0-18.0)$

$4.1 \pm 1.3$

46 (35.7)

110 (85.3)

$115(89.1)$

58 (45.0)

29 (43.3)

$394.7 \pm 98.3$

$59.2 \pm 23.2$

$368.0 \pm 135.8$

$4.1 \pm 1.4$

$33(53.2)$

$50(80.6)$

61 (98.4)

33 (53.2)
TA-APAH

16

$39.1 \pm 9.4$

$13(81.3)^{\pi}$

$54.0(16.0-110.8)^{++}$

$68.0(49.0-91.3)$

$11.0(8.6-18.5)$

$$
7 \text { (43.8) }
$$

$375.1 \pm 139.8$

$58.9 \pm 9.3$

$318.0 \pm 125.0$

$80.0(52.0-98.0)$

$16.09(12.3-22.9)$

$4.0 \pm 1.5$

$0(0)^{\S \S}$

$16(100)$

$10(62.5)^{f f}$

$2(12.5)^{\# \# \#}$
SSC-APAH

8

$39.1 \pm 13.7$

$7(87.5)^{\oplus \%}$

$12.3(9.8-33.0)$

5 (62.5)

$436.4 \pm 41.2$

$67.9 \pm 25.4$

$361.3 \pm 133.0$

$100.0(49.0-112.0)$

11.5 (8.0-13.7)

$4.2 \pm 1.6$

4 (50.0)

7 (87.5)

$6(75)^{+++}$

$2(25.0)^{\S \S \S}$

Data are presented as mean \pm SD, $\mathrm{n}(\%)$ or median (interquartile range), unless otherwise stated. SLE: systemic lupus erythematosus; APAH; associated pulmonary arterial hypertension; TA: Takayasu arteritis; SSc: systemic sclerosis; WHO FC: World Health Organization functional class; SCr: serum creatinine; UA: uric acid; ALP: alkaline phosphatase; TBil: total bilirubin; TC: total cholesterol; RNP: ribonucleoprotein. \#: duration from first pulmonary arterial hypertension (PAH)-related symptoms (physical activity-caused dyspnoea, fatigue, chest pain or near syncope) to PAH diagnosis by right heart catheterisation; ${ }^{\circ}$ : normal range $44-133 \mu \mathrm{mol} \cdot \mathrm{L}^{-1} ;{ }^{+}$: normal range $150-420 \mu \mathrm{mol} \cdot \mathrm{L}^{-1}$; ${ }^{\S}$ : normal range $40-160 \mathrm{IU} \cdot \mathrm{L}^{-1}$; ${ }^{f}$ : normal range $1.7-20 \mu \mathrm{mol} \cdot \mathrm{L}^{-1} ;{ }^{\# \#}$ : normal range $3.2-5.1 \mathrm{mmol} \cdot \mathrm{L}^{-1} ;{ }^{~ " \%}: \mathrm{p}=0.028$ (TA-APAH versus SLE-APAH); ${ }^{++}: \mathrm{p}=0.002($ TA-APAH versus SLE-APAH); ${ }^{\S \S}: p=0.001$ (TA-APAH versus SLE-APAH); ${ }^{f f}: p<0.0001$ (TA-APAH versus SLE-APAH); ${ }^{\# \# \#: ~} p=0.014$ (TA-APAH versus SLE-APAH); "\%: $p=0.043$ (SSc-APAH versus SLE-APAH); ${ }^{+++}: p=0.009$ (SSc-APAH versus SLE-APAH); ${ }^{\S \S}: p=0.004$ (SSc-APAH versus SLE-APAH). 
TABLE 2 Baseline cardiac and pulmonary investigation parameters of all patients and three connective tissue disease subgroups

\begin{tabular}{|c|c|c|c|c|}
\hline & All patients & SLE-APAH & TA-APAH & SSC-APAH \\
\hline Patients n & 129 & 62 & 16 & 8 \\
\hline \multicolumn{5}{|l|}{ TTE results } \\
\hline sPAP mmHg & $89.3 \pm 23.4$ & $87.8 \pm 221.4$ & $79.8 \pm 30.4$ & $85.5 \pm 29.0$ \\
\hline RVDD mm & $35.7 \pm 11.8$ & $36.8 \pm 11.7$ & $39.6 \pm 11.3$ & $31.0 \pm 9.2$ \\
\hline PCE & $32(24.8)$ & $16(29.6)$ & $4(26.7)$ & $2(25.0)$ \\
\hline \multicolumn{5}{|l|}{ PFT results } \\
\hline FVC $\%$ pred & $82.9 \pm 13.0$ & $84.4 \pm 12.6$ & $80.8 \pm 14.8$ & $80.4 \pm 12.5$ \\
\hline DLCo \% pred & $67.6 \pm 21.3$ & $69.8 \pm 18.3$ & $74.0 \pm 18.4$ & $65.8 \pm 32.7$ \\
\hline FVC/DLCO & $1.36 \pm 0.57$ & $1.31 \pm 0.52$ & $1.14 \pm 0.29$ & $1.62 \pm 1.22$ \\
\hline \multicolumn{5}{|l|}{ RHC results } \\
\hline mRAP $\mathrm{mmHg}$ & $7.2 \pm 4.5$ & $7.2 \pm 4.8$ & $8.3 \pm 5.7$ & $6.1 \pm 4.5$ \\
\hline mPAP mmHg & $51.1 \pm 14.3$ & $49.6 \pm 11.9$ & $47.6 \pm 12.8$ & $50.7 \pm 13.5$ \\
\hline Cardiac index $\mathrm{L} \cdot \mathrm{min}^{-1} \cdot \mathrm{m}^{-2}$ & $2.7 \pm 0.9$ & $2.8 \pm 0.9$ & $2.6 \pm 1.0$ & $2.7 \pm 0.7$ \\
\hline PVR Wood units & $10.6(7.1-16.0)$ & $9.8(7.2-15.2)$ & $9.4(5.4-17.4)$ & $11.1(4.4-14.6)$ \\
\hline $\mathrm{SvO}_{2} \%$ & $61.8 \pm 10.9$ & $62.3 \pm 12.2$ & $59.6 \pm 10.9$ & $63.7 \pm 6.1$ \\
\hline \multicolumn{5}{|c|}{$\begin{array}{l}\text { Data are presented as mean } \pm \text { SD, } n(\%) \text { or median (interquartile range), unless otherwise stated. SLE: systemic lupus erythematosus; APAH: } \\
\text { associated pulmonary arterial hypertension; TA: Takayasu arteritis; SSc: systemic sclerosis; TTE: transthoracic echocardiography; sPAP: systolic } \\
\text { pulmonary artery pressure; RVDD: right ventricular diastolic diameter; PCE: pericardial effusion; PFT: pulmonary function test; FVC: forced vital } \\
\text { capacity; DLCo: diffusing capacity of the lung for carbon monoxide; RHC: right heart catheterisation; mRAP: mean right atrial pressure; mPAP: } \\
\text { mean pulmonary arterial pressure; PVR: pulmonary vascular disease.; } \mathrm{SvO}_{2} \text { : mixed venous oxygen saturation. }\end{array}$} \\
\hline
\end{tabular}

\section{Survival analysis}

15 patients were lost to follow-up. The remaining114 patients were followed up for a median of 17.3 months (range 1.4-55.1 months), during which 17 (14.9\%) patients died. Most of them died of chronic right heart failure except for three who suffered a sudden death and one who committed suicide. The overall survival rates at 1 and 3 years were $92 \%$ and $80 \%$, respectively. Among the CTD subgroups, the survival rates at 1 and 3 years were $91 \%$ and $88 \%$ for SLE, $100 \%$ and $86 \%$ for pSS, $84 \%$ and $72 \%$ for TA, $82 \%$ and $64 \%$ for MCTD, and $100 \%$ and $60 \%$ for SSc patients, respectively.

Kaplan-Meier analysis revealed no significant differences in survival among PAH patients secondary to the different CTDs. A comparison of survival between SLE-APAH and non-SLE-APAH patients also indicated no significant difference (fig. 2).

In patients with ILD-PH, survival rates at 1 and 3 years were $74 \%$ and 52\%, respectively. Kaplan-Meier analysis showed that the survival of patients with ILD-PH was significantly worse than that of isolated CTDAPAH patients $(\mathrm{p}<0.05)$ (fig. 3).

\section{Predictors of mortality}

Univariate Cox analysis showed that patients with pericardial effusion and higher PVR and alkaline phosphatase (ALP) values had a higher risk of death (all $\mathrm{p}<0.05)$, as did patients with lower total cholesterol (TC) $(\mathrm{p}<0.05)$. However, patients with higher $6 \mathrm{MWD}$ and $\mathrm{SvO}_{2}$ values had a lower risk of death (both $\mathrm{p}<0.05$ ). WHO FC III/IV and a lower cardiac index also tended to be associated with a higher risk of death, but the differences were not statistically significant. Multivariable analysis showed that higher PVR and ALP values were both independent predictors of survival. Each additional 3 Wood units of PVR and $50 \mathrm{IU} \cdot \mathrm{L}^{-1}$ of ALP would, respectively, lead to 1.3 and 1.7 times greater risks to death. The effects of age, sex, duration from onset of symptoms to diagnosis of $\mathrm{PAH}$, autoantibodies and medications on survival were also tested, but all appeared to be insignificant by either univariate or multivariate analysis (table 3 ).

\section{Discussion}

This study represents the largest series of Chinese patients with CTD-APAH confirmed by RHC to be reported thus far. As expected, the most important finding of this study was that SLE was the most common underlying CTD, while the second and third most common CTDs were pSS and TA. In contrast to Caucasian cohorts, only $8 \%$ of our patients had SSc-APAH. The epidemiology of CTDs in Chinese and Caucasian patients may explain the difference. SLE is much more common in Chinese persons than Caucasians. Most of epidemiological studies of rheumatic diseases in Chinese population showed the 


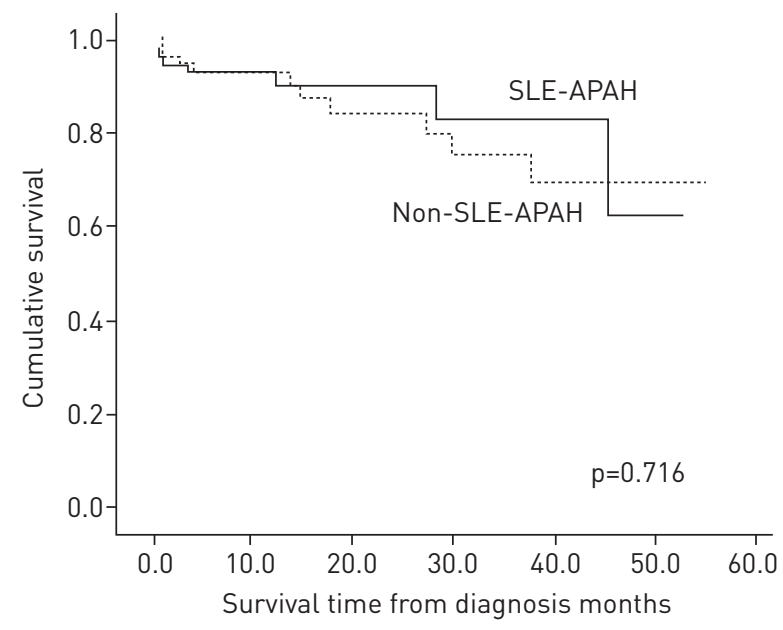

$\begin{array}{rrrccc}\text { SLE-APAH n } 55 & 40 & 20 & 11 & 7 & 1 \\ \text { Non-SLE-APAH n } 59 & 40 & 27 & 18 & 10 & 4\end{array}$

FIGURE 2 Kaplan-Meier analysis of survival in systemic lupus erythematosus (SLE)-associated pulmonary arterial hypertension (APAH) and other connective tissue disease-APAH (non-SLE-APAH) patients. There was no significant difference in survival between the two groups. The 1- and 3-year survival rates for the SLE-APAH group were 91\% and 88\%, and for non-SLE-APAH group they were $92 \%$ and $75 \%$, respectively.

estimated prevalence of SLE was 50-100 cases per 100000 persons [17-19], but most of the data from Europe and North America showed the prevalence of SLE in Caucasians was 10-35 per $100000[4,20]$. Conversely, the prevalence of SSc was higher in Caucasian (8.8-30.5 per 100000) $[7,8,21,22]$ than in Chinese (6.5-10 per 100 000) populations [9, 23]. A retrospective study conducted at one of the largest rheumatology centres in China found that $60.6 \%$ of 2189 CTD in-patients seen over a 7 -year period were SLE patients, but only $3.2 \%$ had SSc [24]. At one of the centres involved in the present study (Rheumatology Dept, Peking University First Hospital, Beijing), the SLE patients account for $18.8 \%$ of 2127 outpatients who have been regularly followed up during the last 5 years, but SSc patients account for only $1.9 \%$ of all patients (unpublished observations). It is noteworthy that this rheumatology centre is not a lupus-specialised centre. Since patients with all types of rheumatic diseases are seen at this centre, these data should reflect the distribution of patients with CTD-APAH throughout China.

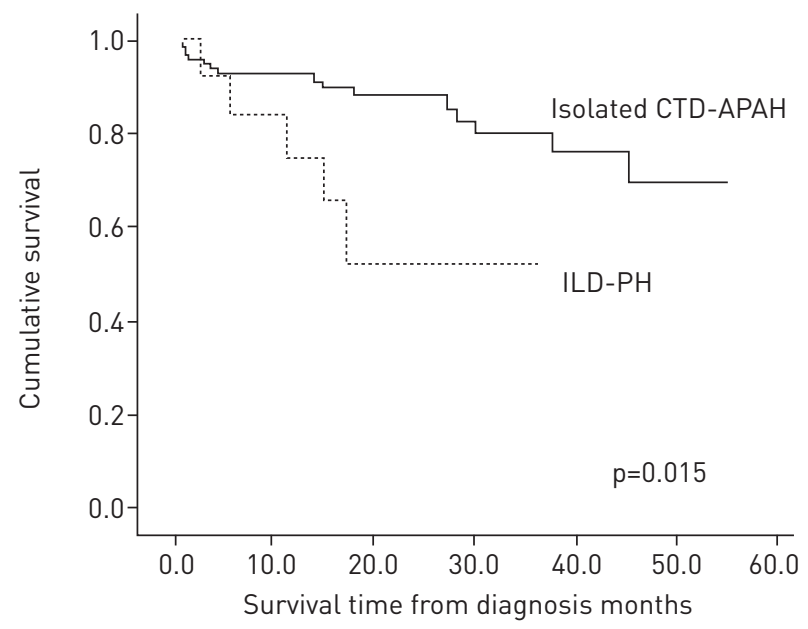

$\begin{array}{rcccccc}\begin{array}{r}\text { Isolated } \\ \text { CTD-APAH n }\end{array} & 114 & 79 & 50 & 29 & 17 & 5 \\ \text { ILD-PH n } & 15 & 10 & 3 & 1 & \end{array}$

FIGURE 3 Kaplan-Meier analysis of survival in connective tissue disease (CTD) patients with interstitial lung diseaseassociated pulmonary hypertension (ILD- PH) and isolated CTD-associated pulmonary arterial hypertension (APAH) patients. The survival of ILD-PH patients was significantly worse than that of isolated CTD-APAH patients. The 1- and 3-year survival rates for the ILD-PH group were $74 \%$ and $52 \%$, and for the isolated CTD-APAH group they were $92 \%$ and $80 \%$, respectively. 
TABLE 3 Predictors for mortality by Cox regression analysis

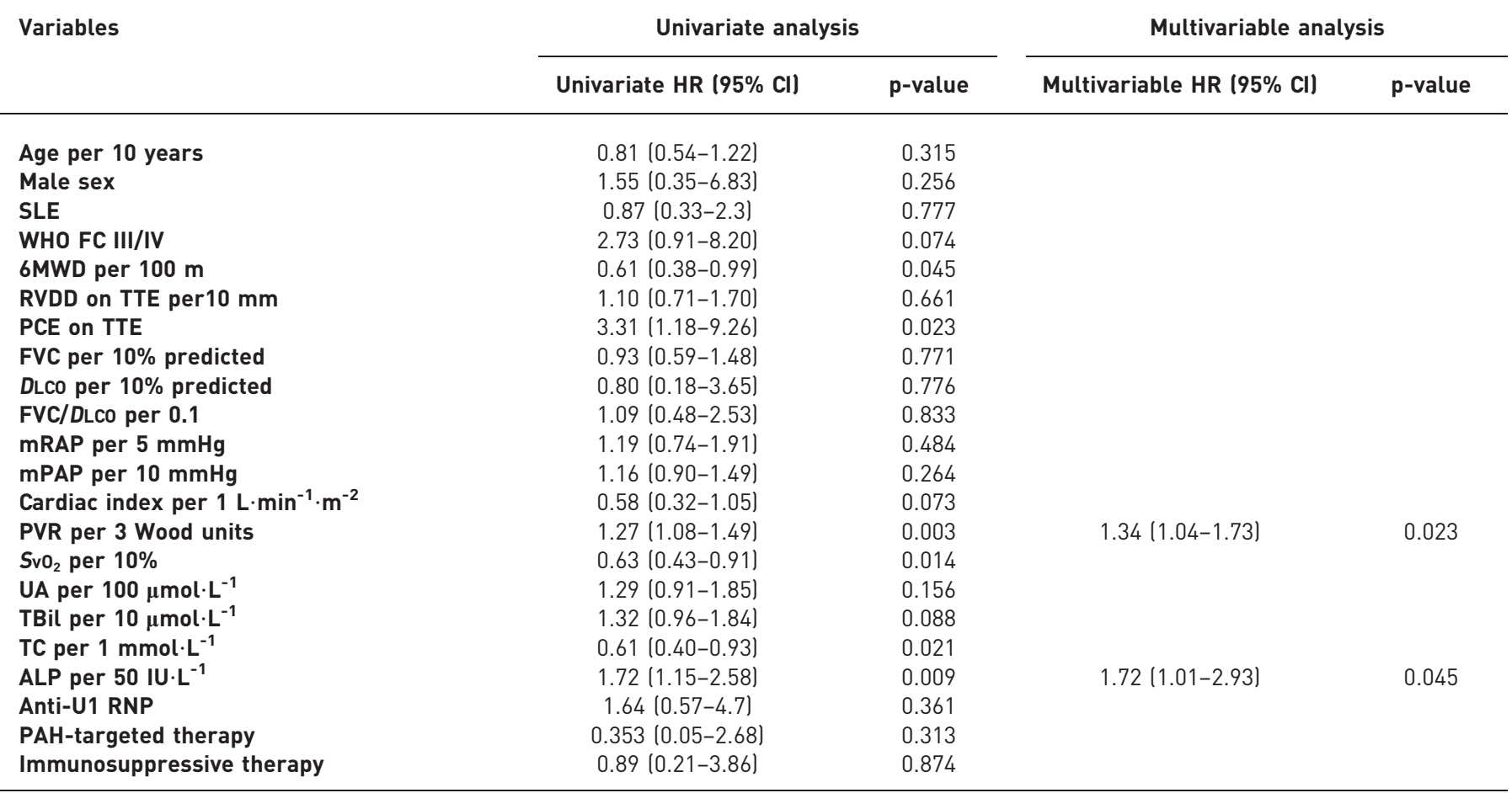

HR: hazard ratio; SLE: systemic lupus erythematosus; WHO FC: World Health Organization functional class; 6MWD: 6-min walk distance; RVDD: right ventricular diastolic diameter; TTE: transthoracic echocardiography; PCE: pericardial effusion; FVC: forced vital capacity; DLCO: diffusing capacity of the lung for carbon monoxide; mRAP: mean right atrial pressure; mPAP: mean pulmonary artery pressure; PVR: pulmonary vascular disease; $\mathrm{SvO}_{2}$ : mixed venous oxygen saturation; UA: uric acid; TBil: total bilirubin; TC: total cholesterol; ALP: alkaline phosphatase; RNP: ribonucleoprotein; PAH: pulmonary arterial hypertension.

As with SLE, pSS is also common in China [18], and TA is considered more common in China and other East Asian countries than in western countries [25]. A Japanese study reported that the prevalence of TA was four per 100000 [26] and a small-scale survey in China indicated the prevalence of TA was 13 per 100000 [27]. In contrast, some epidemiology studies have shown that the prevalence of TA in European countries was only $0.47-0.95$ per 100000 [28]. Interestingly, there were three patients with adult-onset Still's disease complicating PAH in our cohort, which has rarely been reported before [29].

TA, although not strictly considered as an aetiology of CTD-PAH, was included in our registry because it is highly prevalent in China and can be complicated by PAH. Unlike other CTD-APAH, in which the lesions are mainly located in medium to small pulmonary arterioles, TA-APAH most frequently affects the main, lobar and/or segmental pulmonary arteries [30-34]. However, some research indeed demonstrated that distal small pulmonary arterioles could be involved in TA [34] and the plexiform lesion that was regarded as typical of PAH was also observed in the surgical specimens from TA patients [35]. In our study, we observed that TA-APAH patients had a higher sPAP and lower dPAP than other CTD-APAH patients. This variation may be correlated with the difference in pathogenesis. As a special phenotype, TA-APAH definitely requires further study.

Compared to other studies from the USA and Europe in the modern management era, such as the Registry to Evaluate Early and Long-Term PAH Disease Management (REVEAL) from the USA, the national registry study from UK and the national prospective study from France $[1,2,36]$, the survival rates of overall CTDAPAH, SLE-APAH, MCTD-APAH and SSc-APAH patients in our study were not inferior (table 4). The 1and 3-year survival rates of our patients with SLE-APAH were even superior to those of the UK study, which ended before 2007 [2]. The improvement may correlate with further increased attention to CTDAPAH and more appropriate use of medications targeting PAH in recent years.

The UK study first showed the survival of SLE-APAH was better than SSc-APAH [2]. This trend was also found in our cohort, although the difference was not statistically significant, probably due to the small number of SSc-APAH patients. Some previous studies have suggested that some patients with SLE-APAH 
TABLE 4 Comparisons of survival rates among different group of patients from different studies

\begin{tabular}{|c|c|c|c|c|c|c|c|}
\hline & $\begin{array}{c}\text { Overall } \\
1 \text { year }\end{array}$ & \multicolumn{2}{|c|}{ SLE-APAH } & \multicolumn{2}{|c|}{ MCTD-APAH } & \multicolumn{2}{|c|}{ SSC-APAH } \\
\hline REVEAL [1] & 86 & 94 & NA & 88 & NA & 82 & NA \\
\hline CondLiffe et al. [2] & NA & 78 & 74 & 89 & 63 & 78 & 47 \\
\hline LAUNAY et al. [30] & NA & NA & NA & NA & NA & 90 & 56 \\
\hline
\end{tabular}

Data are presented as \%. SLE: systemic lupus erythematosus; APAH: associated pulmonary arterial hypertension; MCTD: mixed connective tissue disease; SSc: systemic sclerosis; REVEAL: Registry to Evaluate Early and Long-Term Pulmonary Arterial Hypertension Disease Management; NA: not available.

respond to immunosuppressive therapy; however, SSc-APAH patients did not $[37,38]$. The differences in therapeutic response and prognosis reflect the variations of pathological mechanisms between the two types of CTD-APAH. To improve the poor prognosis of SSc-APAH, some useful tools for early screening of PAH in SSc patients, for instance, tricuspid regurgitation velocity, $\mathrm{N}$-terminal pro-brain natriuretic peptide and pulmonary function tests, have been developed [39-42], and early screening and management have been recommended and adopted in clinical practice in Europe, North America and Australia [39, 43].

The survival of CTD patients with ILD-PH was significantly worse than that of isolated CTD-APAH patients in our study. Three previous reports from the UK, USA and France obtained the same results as ours when comparing the survival of ILD-PH or respiratory-associated $\mathrm{PH}$ with isolated PAH in SSc patients $[2,44,45]$. One possible explanation for the poorer prognosis of ILD-PH patients is remarkably reduced oxygen diffusion function due to interstitial lesion itself, which can lead to worsened hypoxaemia. In our study, the DLCO \% predicted in ILD-PH group was significantly lower than the isolated CTD-APAH group $(55.7 \pm 21.5 \%$ versus $67.6 \pm 21.3 \%, \mathrm{p}<0.05)$, while the oxygen use rate at baseline was much higher (34.5\% in the ILD-PH group versus $19.1 \%$ in the CTD-APAH group, $\mathrm{p}<0.05$ ). The studies from both the USA and France also found similar results. Meanwhile, they showed a decreased DLCO was correlated with reduced survival in patients with ILD-PH $[44,45]$.

Pericardial effusion, PVR, 6MWD and $\mathrm{SvO}_{2}$ were associated with prognosis in our CTD-APAH patients, which is consistent with several previous studies [2, 46, 47]. Among these parameters, PVR was identified as an independent risk factor for mortality in our study, but mPAP was not. Our finding supports PVR being a better and more important prognostic marker than PAP in patients with CTD-APAH. In addition to functional and haemodynamic markers, serological ALP and TC were shown to be associated with prognosis, and ALP was an independent risk factor for mortality, which has rarely been reported. Liver dysfunction due to heart failure was reported to be characterised by a cholestatic profile. Elevation of ALP, $\gamma$-glutamyl transpeptidase and bilirubin are common abnormalities in congestive cardiac hepatopathy, and have been found to be associated with the severity of tricuspid regurgitation and sPAP estimated by echocardiography, but not elevation of alanine aminotransferase and aspartate transaminase [48]. As cholesterol is synthesised in liver, hypolipidaemia can be present in liver dysfunction. Therefore, ALP and TC, which probably reflect the severity of congestive cardiac hepatopathy, are reasonable as being prognostic biomarkers. In our study, we did not find the influence of immunosuppressive therapy on survival, which may be due to the study's retrospective nature, the diverse doses of glucocorticoids and the diverse types of immunosuppressants.

There are some limitations of this study. Firstly, as a retrospective, observational study, the ability to avoid data bias was limited. Secondly, comparisons between different CTDs were hampered by the small numbers of patients with some CTD types. Thirdly, the survival time was calculated from the time of diagnostic RHC (due to difficulties in determining the precise disease onset time), which may influence the survival analysis.

In summary, our findings indicate that SLE is the most common underlying disease of CTD-APAH in Chinese patients. This is in contrast to reports from western countries in which SSc-APAH was the most common type. Our data suggest that the overall short-term survival of Chinese patients with CTD-APAH has been improved in the modern treatment era and is currently similar to that of western countries. Higher PVR and ALP values are associated with a higher risk of death, as is a lower TC value. Notably, elevated PVR and ALP are independent risk factors for mortality. However, higher 6MWD and $\mathrm{SvO}_{2}$ are associated with a lower risk of death. 


\section{References}

1 Chung L, Liu J, Parsons L, et al. Characterization of connective tissue disease-associated pulmonary arterial hypertension from REVEAL. Identifying systemic sclerosis as a unique phenotype. Chest 2010; 138: 1383-1394.

2 Condliffe R, Kiely DG, Peacock AJ, et al. Connective tissue disease-associated pulmonary arterial hypertension in the modern treatment era. Am J Respir Crit Care Med 2009; 179: 151-157.

3 Lau CS, Yin G, Mok MY. Ethnic and geographical differences in systemic lupus erythematosus: an overview. Lupus 2006; 15: 715-719.

4 Danchenko N, Satia JA, Anthony MS. Epidemiology of systemic lupus erythematosus: a comparison of worldwide disease burden. Lupus 2006; 15: 308-318.

5 Osio-Salido E, Manapat-Reyes H. Epidemiology of systemic lupus erythematosus in Asia. Lupus 2010; 19: 1365-1373.

6 Barnes J, Mayes MD. Epidemiology of systemic sclerosis: incidence, prevalence, survival, risk factors, malignancy, and environmental triggers. Curr Opin Rheumatol 2012; 24: 165-170.

7 Chifflot H, Fautrel B, Sordet C, et al. Incidence and prevalence of systemic sclerosis: a systemic literature review. Semin Arthritis Rheum 2008; 37: 223-235.

8 Le Guern V, Mahr A, Mouthon L, et al. Prevalence of systemic sclerosis in a French multi-ethnic country. Rheumatology (Oxford) 2004; 43: 1129-1137.

9 Kuo CF, See LC, Yu KH, et al. Epidemiology and mortality of systemic sclerosis: a nationwide population study in Taiwan. Scand J Rheumatol 2011; 40: 373-378.

10 Galiè N, Hoeper MM, Humbert M, et al. Guidelines for the diagnosis and treatment of pulmonary hypertension: the Task Force for the Diagnosis and Treatment of Pulmonary Hypertension of the European Society of Cardiology (ESC) and the European Respiratory Society (ERS), endorsed by the International Society of Heart and Lung Transplantation (ISHLT). Eur Heart J 2009; 30: 2493-2537.

11 Tan EM, Cohen AS, Fries JF, et al. The 1982 revised criteria for the classification of systemic lupus erythematosus. Arthritis Rheum 1982; 25: 1271-1277.

12 Hochberg MC. Updating the American College of Rheumatology revised criteria for the classification of systemic lupus erythematosus. Arthritis Rheum 1997; 40: 1725.

13 Vitali C, Bombardieri S, Jonsson R, et al. Classification criteria for Sjögren's syndrome: a revised version of the European criteria proposed by the American-European Consensus Group. Ann Rheum Dis 2002; 61: 554-558.

14 Arend WP, Michel BA, Bloch DA, et al. The American College of Rheumatology 1990 criteria for the classification of Takayasu arteritis. Arthritis Rheum 1990; 33: 1129-1134.

15 Sharp GC. Diagnostic criteria for classification of MCTD. In: Kasukawa R, Sharp GC, eds. Mixed Connective Tissue Disease and Antinuclear Antibodies. Amsterdam, Excerpta Medica, 1987; pp. 23-30.

16 American Rheumatism Association. Preliminary criteria for the classification of systemic sclerosis (scleroderma). Subcommittee for scleroderma criteria of the American Rheumatism Association Diagnostic and Therapeutic Criteria Committee. Arthritis Rheum 1980; 23: 581-590.

17 Mok CC, Lau CS. Lupus in Hong Kong Chinese. Lupus 2003; 12: 717-722.

18 Zeng QY, Chen R, Darmawan J, et al. Rheumatic diseases in China. Arthritis Res Ther 2008; 10: R17-R27.

19 Mok CC. Epidemiology and survival of systemic lupus erythematosus in Hong Kong Chinese. Lupus 2011; 20: 767-771.

20 Lim SS, Bayakly AR, Helmick CG, et al. The incidence and prevalence of systemic lupus erythematosus, 2002-2004: the Georgia Lupus Registry. Arthritis Rheumatol 2014; 66: 357-368.

21 Andreasson K, Saxne T, Berqknut C, et al. Prevalence and incidence of systemic sclerosis in southern Sweden: population-based data with case ascertainment using the 1980 ARA criteria and proposed ACR-EULAR classification criteria, Ann Rheum Dis 2013 [In press DOI: 10.1136/annrheumdis-2013-203618].

22 Mayes MD, Lacey JV Jr, Beebe-Dimmer J, et al. Prevalence, incidence, survival, and disease characteristics of systemic sclerosis in a large US population. Arthritis Rheum 2003; 48: 2246-2255.

23 Li R, Sun J, Ren LM, et al. Epidemiology of eight common rheumatic diseases in China: a large-scale cross-sectional survey in Beijing. Rheumatology (Oxford) 2012; 51: 721-729.

24 Ji YQ, Zhang ZL, Lu WX. [The clinical analysis of pulmonary arterial hypertension in connective tissue disease]. Chin J Intern Med 2006; 45: 467-471.

25 Kobayashi Y, Numano F. Takayasu arteritis. Intern Med 2002; 41: 44-46.

26 Toshihiko N. Current status of large and small vessel vasculitis in Japan. Int J Cardiol 1996; 54: Suppl., S91-S98.

27 Ren LX, Zhang FS, Yang YH, et al. [The prevalence report of Takayasu arteritis in the northern forest region of China]. J Harbin Med U 1992; 2: 116-117.

28 Watts R, Al-Taiar A, Mooney J, et al. The epidemiology of Takayasu arteritis in the UK. Rheumatology (Oxford) 2009; 48: 1008-1011.

29 Thakare M, Habibi S, Agrawal S, et al. Pulmonary arterial hypertension complicating adult-onset Still's disease. Clin Rheumatol 2013; 32: Suppl. 1, S1-S2.

30 Kasukawa R. Pulmonary hypertension in connective tissue disease. Intern Med 1996; 35: 1-2.

31 Galiè N, Manes A, Farahani KV, et al. Pulmonary arterial hypertension associated to connective tissue diseases. Lupus 2005; 14: 713-717.

32 Pope J. An update in pulmonary hypertension in systemic lupus erythematosus - do we need to know about it? Lupus 2008; 17: 274-277.

33 Proudman SM, Stevens WM, Sahhar J, et al. Pulmonary arterial hypertension in systemic sclerosis: the need for early detection and treatment. Intern Med J 2007; 37: 485-494.

34 Biétry O, Kieffer E, Herson S, et al. Formes artérielles pulmonaires graves de l'artérite de Takayasu. Trois observations et revue de la littérature [Severe pulmonary artery involvement of Takayasu arteritis. 3 cases and review of the literature]. Ar Mal Coeur Vaiss 1991; 84: 817-822.

35 Lie JT. Isolated pulmonary Takayasu arteritis: clinicopathologic characteristics. Mod Pathol 1996; 9: 469-474.

36 Launay D, Sitbon O, Hachulla E, et al. Survival in systemic sclerosis-associated pulmonary arterial hypertension in the modern management era. Ann Rheum Dis 2013; 72: 1940-1946.

37 Jasi X, Launay D, Yaici A, et al. Immunosuppressive therapy in lupus- and mixed connective tissue diseaseassociated pulmonary arterial hypertension: a retrospective analysis of twenty-three cases. Arthritis Rheum 2008; 58: 521-531. 
Sanchez O, Sitbon O, Jais X, et al. Immunosuppressive therapy in connective tissue diseases-associated pulmonary arterial hypertension. Chest 2006; 130: 182-189.

39 Humbert M, Yaici A, de Groote P, et al. Screening for pulmonary arterial hypertension in patients with systemic sclerosis: clinical characteristics at diagnosis and long-term survival. Arthritis Rheum 2011; 63: 3522-3530.

40 Mukerjee D, St George D, Knight C, et al. Echocardiography and pulmonary function as screening tests for pulmonary arterial hypertension in systemic sclerosis. Rheumatology (Oxford) 2004; 43: 461-466.

41 Thakkar V, Stevens MW, Prior D, et al. N-terminal pro-brain natriuretic peptide in a novel screening algorithm for pulmonary arterial hypertension in systemic sclerosis: a case-control study. Arthritis Res Ther 2012; 14: R143-R153.

42 Coghlan JG, Denton CP, Grünig E, et al. Evidence-based detection of pulmonary arterial hypertension in systemic sclerosis: the DETECT study. Ann Rheum Dis 2014; 73: 1340-1349.

43 Galiè N, Rubin LJ, Hoeper MM, et al. Treatment of patients with mildly symptomatic pulmonary arterial hypertension with bosentan (EARLY study): a double-blind, randomised controlled trial. Lancet 2008; 371: 2093-2100.

44 Mathai SC, Hummers LK, Champion HC, et al. Survival in pulmonary hypertension associated with the scleroderma spectrum of diseases: impact of interstitial lung disease. Arthritis Rheum 2009; 60: 569-577.

45 Launay D, Humbert M, Berezne A, et al. Clinical characteristics and survival in systemic sclerosis-related pulmonary hypertension associated with intersititial lung disease. Chest 2011; 140: 1016-1024.

46 Torbicki A, Kurzyna M, Kuca P, et al. Detectable serum cardiac troponin $\mathrm{T}$ as a marker of poor prognosis among patients with chronic precapillary pulmonary hypertension. Circulation 2003; 108: 844-848.

47 Hoeper MM, Oudiz RJ, Peacock A, et al. End points and clinical trial designs in pulmonary arterial hypertension: clinical and regulatory perspectives. J Am Coll Cardiol 2004; 43: Suppl. S, 48S-55S.

48 Lau GT, Tan HC, Kritharides L. Type of liver dysfunction in heart failure and its relation to the severity of tricuspid regurgitation. Am J Cardiol 2002; 90: 1405-1409. 